\title{
Longitudinal non-cystic fibrosis trends of pulmonary Mycobacterium abscessus disease from 2010 to 2017: spread of the "globally successful clone" in Asia
}

\author{
Aristine Cheng (1) $^{1,2}$, Hsin-Yun Sun ${ }^{1,2}$, Yi-Tzu Tsai ${ }^{1}$, Po-Liang Lu ${ }^{3,4}$, \\ Susan Shin-Jung Lee $\mathbb{1}^{5}$, Yi-Tzu Lee ${ }^{6,7}$, Yung-Chih Wang ${ }^{8}$, Po-Yu Liu ${ }^{9}$, \\ Jung-Yien Chien (10), Po-Ren Hsueh ${ }^{1,2}, 10$, Shu-Yuan Chang ${ }^{1}$, Un-In Wu ${ }^{1,2}$, \\ Wang-Huei Sheng ${ }^{1,2}$, Yee-Chun Chen $^{1,2}$ and Shan-Chwen Chang ${ }^{1,2}$
}

\section{ABSTRACT}

Background: Mycobacterium abscessus (MAB) has emerged as the predominant pulmonary nontuberculous mycobacterial pathogen in parts of Asia, including Taiwan. The reasons for the significant increase in $\mathrm{MAB}$ infections in the non-cystic fibrosis $(\mathrm{CF})$ populations are poorly understood. The study aimed to elucidate whether this increase is related to the spread of the globally successful clone of MAB.

Methods: We performed multilocus sequence typing of 371 nonduplicated MAB pulmonary isolates from 371 patients sampled between 2010-2017 at seven hospitals across Taiwan.

Results: In total, 183 (49.3\%) isolates were M. abscessus subsp. abscessus (MAB-a), 187 (50.4\%) were M. abscessus subsp. massiliense (MAB-m), and $1(0.3 \%)$ was M. abscessus subsp. bolletii (MAB-b). MAB-a sequence type (ST)1 (23.7\%) and ST127 (3.8\%), followed by MAB-m ST48 (16.2\%), ST117 (15.1\%), ST23 $(8.6 \%)$ were most common overall. Of MAB-a strains, 50 (27.3\%) belonged to novel STs and $38(10.2 \%)$ were singleton strains, while of MAB-m strains, only $10(5.3 \%)$ were novel and $8(2.2 \%)$ were singletons. From 2010 to 2017, the frequency of the historically dominant ST1 declined from $28.6 \%$ to $22.5 \%$, whereas the recently emerged globally successful clonal cluster 3, ST23 and ST48, increased from $14.3 \%$ to $40.0 \%$.

Conclusions: The dominance of ST1 particularly in the last 2 years of this study appears to be declining, while ST23, reported in outbreaks among CF and post-surgical cohorts across the Americas and Europe, alongside the closely related ST48, is present among non-CF populations in Taiwan. These trends need to be confirmed with further ongoing studies to track the molecular epidemiology of clinical MAB isolates worldwide.

@ERSpublications

The globally successful clonal cluster 3 of $M$. abscessus is present among non-CF individuals in Taiwan and there is a recent decline in the historically predominant ST1, warranting further study https://bit.ly/3jS2q1n

Cite this article as: Cheng A, Sun H-Y, Tsai Y-T, et al. Longitudinal non-cystic fibrosis trends of pulmonary Mycobacterium abscessus disease from 2010 to 2017: spread of the "globally successful clone" in Asia. ERJ Open Res 2021; 7: 00191-2020 [https://doi.org/10.1183/23120541.00191-2020].

Received: 16 April 2020 | Accepted after revision: 30 Sept 2020

Copyright $\odot$ ERS 2021. This article is open access and distributed under the terms of the Creative Commons Attribution Non-Commercial Licence 4.0. 


\section{Introduction}

Mycobacterium abscessus (MAB) is the commonest rapidly growing non-tuberculous mycobacteria (NTM) causing pulmonary infections worldwide [1-3]. In recent years, MAB has undergone multiple taxonomic revisions [4-8]. However, three closely related entities are recognised: M. abscessus subsp. abscessus (MAB-a), M. abscessus subsp. bolletii (MAB-b) and M. abscessus subsp. massiliense (MAB-m) [6, 7]. They differ in terms of drug susceptibility, and may have differences related to transmissibility [9-11]. Pulmonary infection is the most typical clinical presentation, but extrapulmonary infection either due to direct inoculation into the skin or due to disseminated disease, often in association with neutralising anti-interferon (IFN)- $\gamma$ autoantibodies is recognised with increased frequency [12-14].

Previous studies have indicated great diversity within MAB among cystic fibrosis (CF) patients, suggesting independent acquisitions from the environment $[15,16]$. However, suspicion of patient-to-patient transmission arose after two reports of respiratory outbreaks with MAB-m at different CF centres across the Atlantic $[10,17,18]$. One outbreak occurred in Seattle, USA, wherein the index case patient and four additional patients were infected with near-identical MAB-m isolates with resistance to amikacin and clarithromycin [17]. The second outbreak occurred in the United Kingdom, involving 11 patients who all had MAB-m infections sharing the same constitutive resistance to clarithromycin and amikacin, despite some individuals being naive to long-term macrolide or aminoglycoside therapy [10].

By whole-genome sequencing (WGS), isolates from these two CF centres, were subsequently found to be highly related, belonging to sequence type 23 (ST23) and clonal cluster 3 (CC3) $[18,19]$. Meanwhile, an epidemic of at least 2032 post-surgical infections between 2004-2011 across Brazil was also due to ST23 (CC3), and an outbreak of post-procedural infections between 2010-2012 in Taiwan was due to ST48, differing from ST23 only at the murC locus (also within CC3), thereafter referred to as the "globally successful clone" [20-22].

In parts of Asia including Singapore, Okinawa and southern Taiwan, MAB has overtaken the Mycobacterium avium complex as the most common NTM causing lung disease [23-25]. CF is extremely rare in such populations with an estimated incidence of 3.12 per million live births in the Japanese population and fewer than 1 in 90000 live births among Eastern Asian people [26]. One hypothesis for the rising dominance of $\mathrm{MAB}$ is that evolutionary changes affecting environmental adaptation, transmissibility, and virulence to humans may have enhanced the spread of the globally successful clone (CC3). This clone may not follow traditional patterns of MAB infection and may affect the general population without pre-requisite for abnormal lungs or airways. However, comparable molecular epidemiology studies are lacking in Asia and non-CF populations. Longitudinal population studies are unreported worldwide. Thus, the aim of this study is to investigate the molecular epidemiology of MAB in Taiwan which has not been reported before.

\section{Methods}

Hospital sites

Seven hospitals participated in this nationwide study (figure 1). The institutional ethic committees of the participating sites approved the study (NTUH REC 201605114RIND, KMUHIRB-E(I)-20180008, TSGH REC 2-108-05-113) or waived the need for formal review under the auspices of infection control.

\section{Mycobacterial isolates}

Pulmonary MAB isolates were identified using the hospital laboratory database. Random sampling was performed by date criteria (samples submitted every first and 15th day of each month starting from January 2010 and ending in December 2017) were collected from each hospital site and submitted to the National Taiwan University Hospital, Taipei (site A) for genotypic verification and typing. Only the first $\mathrm{MAB}$ isolate for each patient was included (duplicates were excluded). The maximum number of MAB isolates submitted for genotypic identification by multilocus sequence analysis (MLSA) per year per site

Affiliations: ${ }^{1}$ Dept of Internal Medicine, National Taiwan University Hospital, Taipei, Taiwan. ${ }^{2}$ College of Medicine, National Taiwan University, Taipei, Taiwan. ${ }^{3}$ Dept of Laboratory Medicine, Kaohsiung Medical University Hospital, Kaohsiung. Taiwan. ${ }^{4}$ Graduate Institute of Medicine, College of Medicine, Kaohsiung Medical University, Kaohsiung, Taiwan. ${ }^{5}$ Dept of Internal Medicine, Dept of Pathology and Laboratory, Kaohsiung Veterans General Hospital, Kaohsiung, Taiwan. ${ }^{6}$ Faculty of Medicine, School of Medicine, National Yang-Ming University, Taipei, Taiwan. ${ }^{7}$ Dept of Emergency Medicine, Taipei Veterans General Hospital, Taipei, Taiwan. ${ }^{8}$ Dept of Internal Medicine, Tri-Service General Hospital, National Defense Medical Center, Taipei, Taiwan. ${ }^{9}$ Dept of Internal Medicine, Taichung, Veterans General Hospital, Taichung, Taiwan. ${ }^{10}$ Dept of Laboratory Medicine, National Taiwan University Hospital, Taiwan.

Correspondence: Shan-Chwen Chang, National Taiwan University College of Medicine, No. 1 Ren-Ai Road, Taipei 100, Taiwan. E-mail: changsclantu.edu.tw 
FIGURE 1 Map showing the location of participating hospitals and the included patient numbers at each site Imodified from the Map Taiwan with Counties - Multicolour by FreeVectorMaps.com). NTUH-T: National Taiwan University HospitalTaipei; TSGH: Tri-Service General Hospital; NTUH-HC: National Taiwan University Hospital-Hsinchu; TVGH: Taichung Veterans' General Hospital; NTUH-YL: National Taiwan University Yunlinn; KVGH: Kaohsiung Veterans' General Hospital; KMUH: Kaohsiung Medical University Hospital.

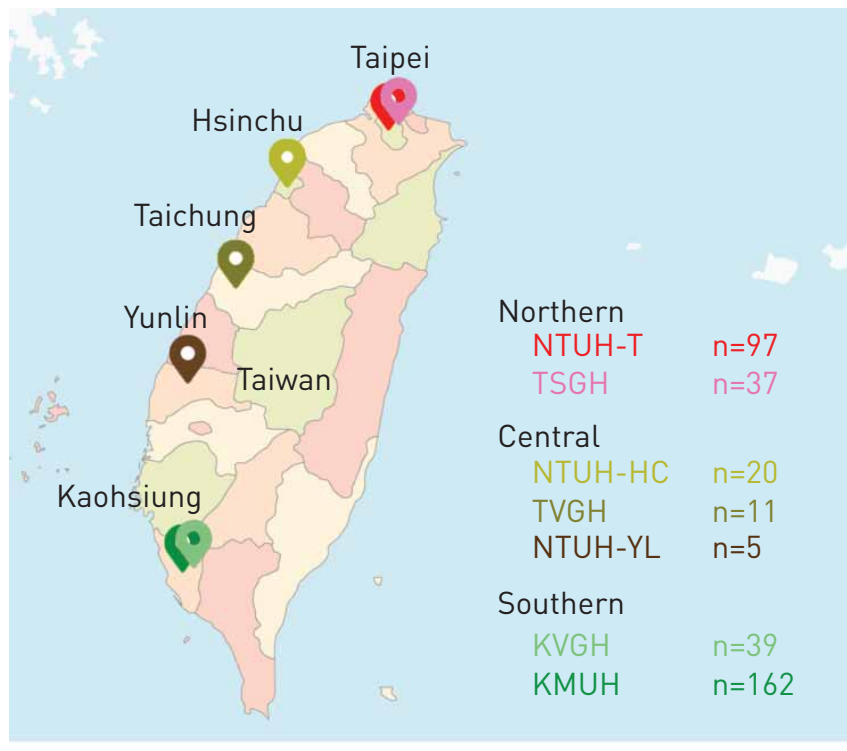

was 35. Excessive isolates per site were randomly excluded by the following method: the first two of every triplet was included (every third isolate was excluded). The maximum number of genotypically confirmed MAB isolates submitted for multilocus sequence typing (MLST) per year per site was 24. All mycobacterial isolates were stored at $-80^{\circ} \mathrm{C}$ in GermBank (Creative Media Products, Wugu Shiang, Taipei County, Taiwan). Immediately prior to use, the strains were subcultured onto sheep blood agar at $35^{\circ} \mathrm{C}$ as described previously [27].

\section{Multilocus sequence analysis}

Molecular confirmation of $\mathrm{MAB}$ isolates was performed by concatenating the partial sequences of three genes (hsp65, rpoB and secA1) according to ZeLAZNY et al. [28]. Only isolates molecularly identified as MAB were included in this study for MLST.

\section{Multilocus sequence typing}

MLST was performed using seven housekeeping genes ( $\operatorname{argH}, c y a, g l p K$, gnd, murC, pta and purH) according to www.bigsdb.pasteur.fr/mycoabscessus/mycoabscessus.html [21]. Phylogenetic trees were constructed using the BioNumerics software (version 6.6; Applied Maths, BioMèrieux, Belgium).

\section{Retrospective chart review}

The clinical relevance of each MAB isolate was determined according to the 2007 ATS/IDSA guidelines [29]. Patient demographics and underlying comorbidities were retrieved. The presence of concomitant respiratory tract pathogens were recorded. All-cause mortality and date of last visit or death were recorded.

\section{Results}

A total of 649 unique MAB pulmonary isolates were retrieved by study criteria from the microbiology laboratory databases of seven hospitals across Taiwan (figures 1 and 2). After excluding isolates in excess of the maximum number of isolates allowed per site by random deletion to avoid over-representation of National Taiwan University Hospital-Taipei, 468 routine microbiological laboratory identified MAB isolates underwent MLSA. Subsequently, 24 were excluded because they were molecularly identified as species other than $\mathrm{MAB}$ and another 73 isolates randomly excluded to avoid over-representation of Kaohsiung Medical University Hospital. Thus, a total of 371 genotypically confirmed MAB isolates were included in this MLST study (figure 2). These strains were isolated from the expectorated sputum $(\mathrm{n}=343)$, endotracheal aspirates $(n=10)$, bronchial washings $(n=10)$, bronchoalveolar lavage specimens $(n=2)$, and biopsied lung tissue $(n=5)$ of 371 patients. Approximately half were men $(n=189,50.9 \%)$ with a median age (interquartile range (IQR)) of 67 (55-77) years (table 1). Patients in this cohort were followed for an average duration of 2.8 years after their cultures yielded $M$. abscessus, during which $30.5 \%(\mathrm{n}=113)$ died. The majority of deaths within hospital were due to severe sepsis, mostly secondary to pneumonia $(n=30$, 45.5\%), and only five were attributed directly to progressive non-tuberculous mycobacterial pulmonary disease $(n=5,7.6 \%)$. Overall, two-thirds of the patients in this cohort $(n=260,70.1 \%)$ were classified as having NTM-pulmonary disease (NTM-PD) by the consensus definitions published in 2007 by the 
Non-duplicated respiratory isolates phenotypically identified as M. abscessus complex between 2010-2017 at 7 hospitals across Taiwan $\mathrm{n}=649$

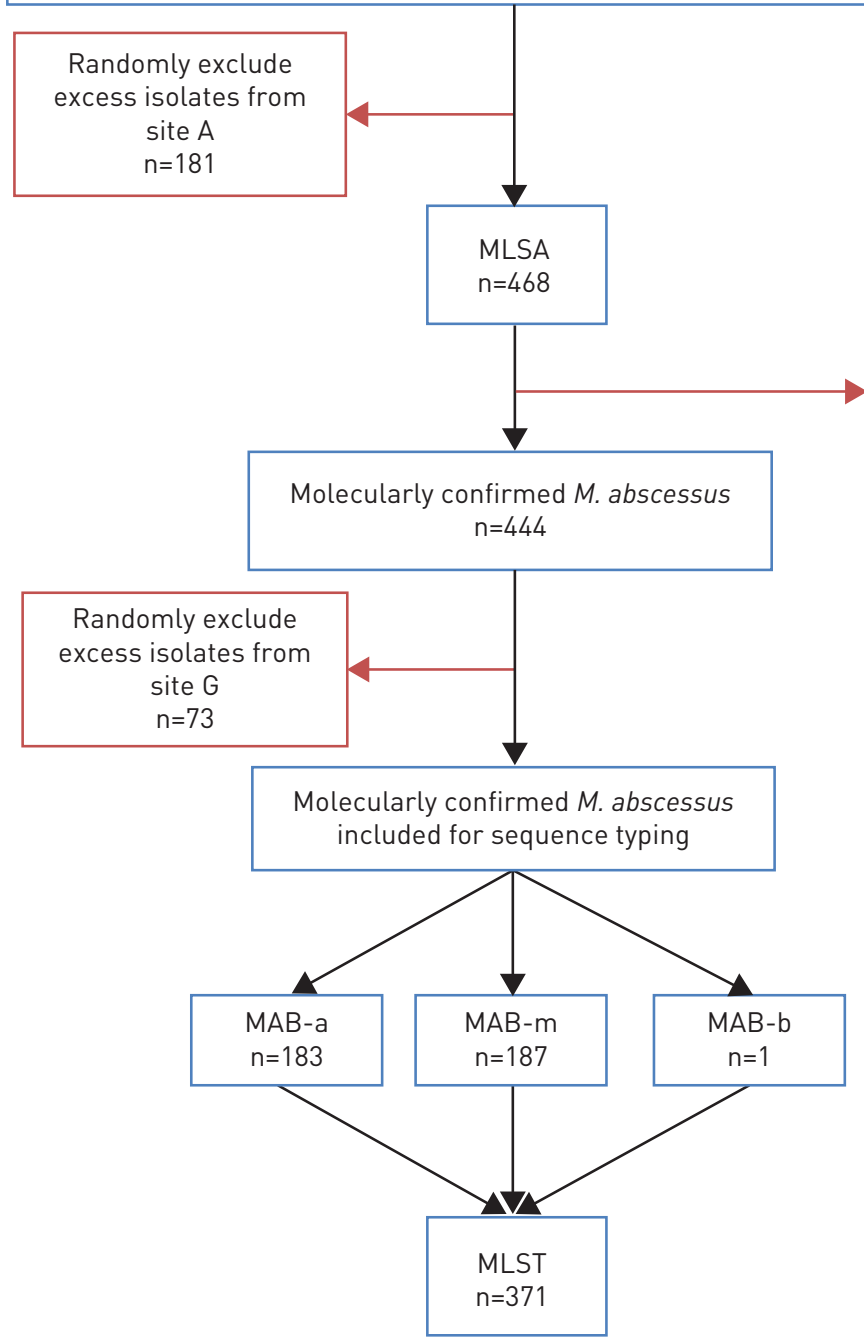

FIGURE 2 Flow diagram of the study. MLSA: multilocus sequence analysis; MAB-a: $M$. abscessus subsp. abscessus; MAB-b: M. abscessus subsp. bolletii; MAB-m: M. abscessus subsp. massiliense.

American Thoracic Society/Infectious Diseases Society of America (ATS/IDSA), of those, 137 (52.7\%) had a nodular-bronchiectatic pattern on computed tomography, 14 (5.4\%) had fibro-cavitary lung disease, and 109 (42.9\%) had a mixed or unclassifiable pattern [29].

The majority of patients had one or more comorbid conditions (table 1). Underlying lung disease was the most common comorbidity $(\mathrm{n}=183,49.3 \%)$, although of note, there were no patients with known CF. The second most common underlying disease was malignancy $(\mathrm{n}=89,24.0 \%)$, notably of the head and neck $(n=22,5.9 \%)$, the upper gastrointestinal tract $(n=16,4.4 \%)$ and the lung $(n=13,3.5 \%)$. However, immunocompromising conditions in the context of susceptibility to mycobacterial infections as listed in table 1, were not overtly common in this cohort of mainly pulmonary and not disseminated disease. Interestingly, a fifth of this elderly population had neurological conditions $(n=76,20.5 \%)$ that often resulted in tracheotomy to avert aspiration, similar to the risks posed by head and neck cancers. The spectrum of neurological disease included ischaemic stroke $(n=28,7.5 \%)$, movement disorders such as amyotrophic lateral sclerosis and Parkinsonism $(\mathrm{n}=19,5.1 \%)$, intracranial haemorrhages $(\mathrm{n}=10,2.7 \%)$, senile dementia $(n=9,2.4 \%)$, brain tumours $(n=5,1.3 \%)$, metabolic or hypoxic encephalopathy $(n=2$, $0.5 \%)$ and other cognitive disorders $(n=2,0.5 \%)$.

Of the 371 MAB isolates, 183 (49.3\%) belonged to the subspecies abscessus (MAB-a) and 187 (50.4\%) belonged to the subspecies massiliense (MAB-m) (table 2). Only one isolate $(0.3 \%)$ was identified as the 
subspecies bolletii (MAB-b). For MAB-a, 55 sequence types (STs) were identified in total, including 48 novel STs (combinations of alleles not matching any of the current 270 STs in the international database) (table 2 and figure 3). The most common MAB-a sequence types was ST1 (comprising $23.7 \%$ of all strains and $48.1 \%$ of all subspecies abscessus strains included in this study), followed by ST127 (3.8\% of all strains and 7.7\% of all MAB-a). There were five isolates each of ST22 (a common ST in Ireland) and ST49 [30].

The 187 strains of MAB-m were assigned to 19 STs, including 7 novel STs. The commonest MAB-m sequence types was ST48 (16.2\% of all strains and $32.1 \%$ of all subspecies massiliense), followed by ST117 (15.1\% overall and $29.9 \%$ of MAB-m), and ST23 (8.6\% overall and $17.1 \%$ of MAB-m). Only 2 MAB-m isolates belonged to ST2, previously reported as the predominant ST in non-outbreak settings among CF patients [21]. A smaller proportion of MAB-m isolates compared to MAB-a isolates (2.2\% versus $10.2 \%)$ were represented in single STs.

\begin{tabular}{|c|c|}
\hline Patients n & 371 \\
\hline Age years & $67(55-77)$ \\
\hline Men & $189(50.9)$ \\
\hline NTM-pulmonary disease & $260(70.1)$ \\
\hline Nodular-bronchiectatic type & $137(36.9)$ \\
\hline Fibro-cavitary type & $14(3.8)$ \\
\hline Mixed or other type & 109 (29.3) \\
\hline Underlying lung diseases & $183(49.3)$ \\
\hline Bronchiectasis & 131 (35.3) \\
\hline Chronic obstructive pulmonary disease & $57(15.4)$ \\
\hline Remote history of pulmonary tuberculosis & $58(15.6)$ \\
\hline Interstitial lung disease & $3(0.8)$ \\
\hline Pneumoconioses & $1(0.3)$ \\
\hline Diabetes mellitus & 72 (19.4) \\
\hline Congestive heart failure & 39 (10.5) \\
\hline Chronic kidney disease stage IV-V & $26(7.0)$ \\
\hline Chronic hepatitis B & $28(7.5)$ \\
\hline Chronic hepatitis C & $11(3.0)$ \\
\hline Autoimmune disease & 18 (4.9) \\
\hline Anti-interferon- $\gamma$ autoantibodies & $9(2.4)$ \\
\hline Anti-GM-CSF autoantibodies & $1(0.3)$ \\
\hline Gastro-oesophageal reflux disease & $43(11.6)$ \\
\hline Human immunodeficiency virus infection & $1(0.3)$ \\
\hline Haematological/bone marrow transplantation & 5 (1.3) \\
\hline Solid organ transplantation & $3(0.8)$ \\
\hline Thyroid disease & $22(5.9)$ \\
\hline Neurological disease & 76 (20.5) \\
\hline Malignancy & $89(24.0)$ \\
\hline Head and neck cancer & $22(5.9)$ \\
\hline Upper gastrointestinal cancer & $16(4.4)$ \\
\hline Lung cancer & $13(3.5)$ \\
\hline Renal, bladder and prostate cancer & $11(3.0)$ \\
\hline Colorectal cancer & $9(2.4)$ \\
\hline Breast cancer & $9(2.4)$ \\
\hline Haematological & 5 (1.3) \\
\hline Neuroendocrine & $3(0.8)$ \\
\hline Other & $4(1.1)$ \\
\hline Deaths during follow-up & $113(30.5)$ \\
\hline In-hospital deaths & $66(17.8)$ \\
\hline Cause of death: sepsis (including pneumonia) & $34(9.2)$ \\
\hline Cause of death: pneumonia & $30(8.1)$ \\
\hline Cause of death: NTM-PD & $5(1.3)$ \\
\hline Cause of death: cancer & $6(1.6)$ \\
\hline Cause of death: haemorrhage & $4(1.1)$ \\
\hline Duration of follow-up years & $2.8 \pm 8.7$ \\
\hline
\end{tabular}

Data are presented as median (interquartile range), $\mathrm{n}(\%)$ or mean $\pm \mathrm{SD}$, unless otherwise stated. NTM: nontuberculous mycobacteria; GM-CSF: granulocyte-macrophage colony-stimulating factor. 
TABLE 2 Mycobacterium abscessus complex isolates from Taiwan (371 isolates from 371 patients)

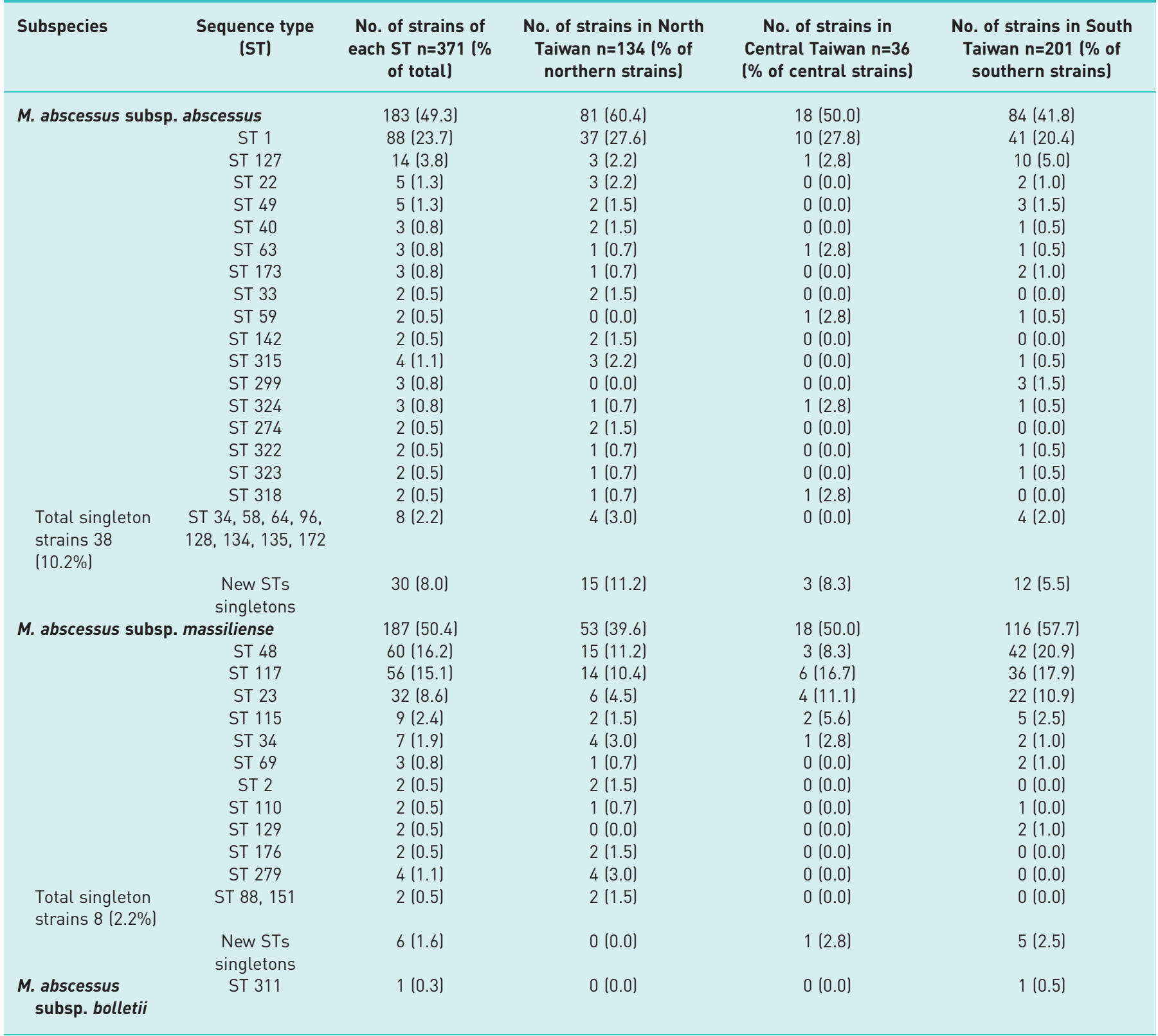

The only isolate of MAB-b in this study had a novel allele at the gnd locus and thus, represented a novel singleton ST.

Geographic distribution of M. abscessus subspecies and sequence types

MAB-a compared to MAB-m was more common in the north (60.4\% versus $39.6 \%$ ), present in equal proportions ( $50 \%$ versus $50 \%$ ) in central Taiwan, and less common in the south ( $41.8 \%$ versus $57.5 \%)$. For MAB-a, ST1 represented $27.6 \%, 27.8 \%$ and $20.4 \%$ of all strains from northern, central and southern Taiwan, respectively, whereas ST127 represented 2.2\%, 2.8\% strains and 5.0\% strains from northern, central and southern Taiwan. For MAB-m, the prevalence of ST48 versus ST23 in northern, central and southern Taiwan were $11.2 \%, 8.3 \%$, and $20.9 \%$, versus $4.5 \%, 11.1 \%$, and $10.9 \%$, respectively, while the prevalence of ST117 gradually increased from north southwards from $10.4 \%$, to $16.7 \%$ and $17.9 \%$.

Changing trends of M. abscessus subspecies and sequence types over time

The frequencies of the common STs for each consecutive year from 2010 to 2017 are shown in table 3 and figure 4. A possible declining trend of ST1 (MAB-a) and ST117 (MAB-m) over the study period $(\mathrm{p}<0.01)$ 
FIGURE 3 Minimum spanning phylogenetic trees derived from multilocus sequence typing (MLST) using the integrated concatenated sequences of seven housekeeping genes: $\operatorname{argH}$, cya, glpK, gnd, murC, pta, and purH (BioNumerics V.6.6, Applied Maths). Strains clustered together by the seven-gene MLST scheme were depicted as circles, strains from different hospitals were depicted by different colours with shades of green representing southern Taiwan, shades of yellow representing central Taiwan, and shades of red representing northern Taiwan.
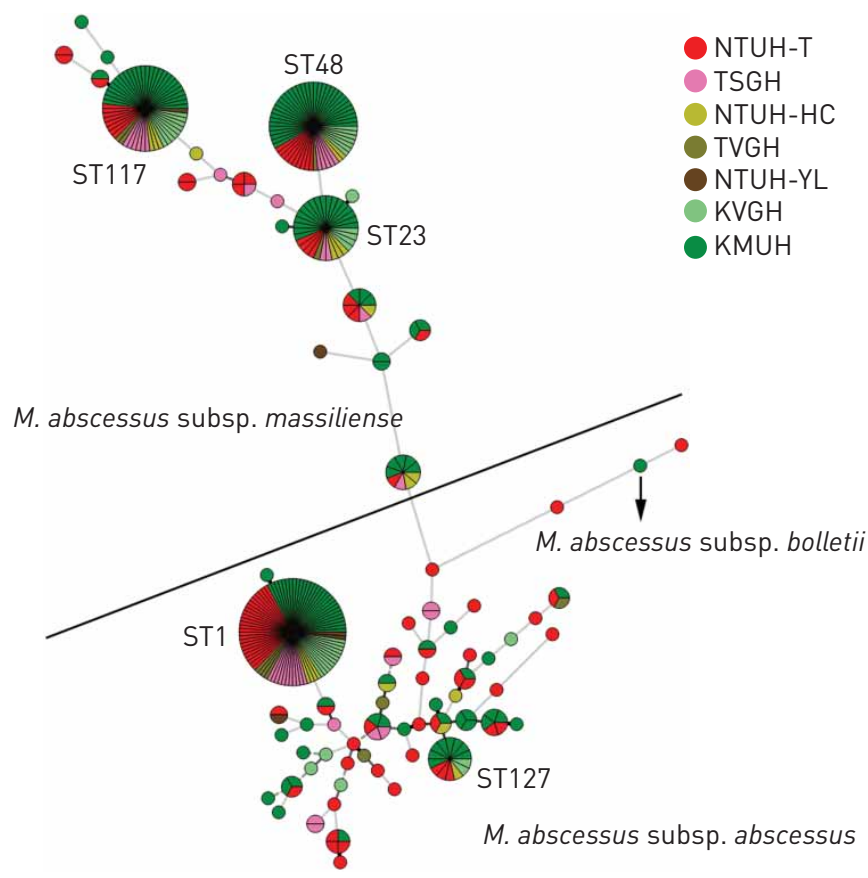

in contrast to a possible increase in the globally successful clone (ST48 and ST23 combined p=0.02) awaits confirmation by further study. ST23 was not detected among northern Taiwan isolates until 2014, whereas ST23 had been present among southern Taiwan isolates since 2010. ST23 was detected in all regions of Taiwan by 2017 .

\section{Clinical descriptions of M. abscessus sequence types}

Patients with ST23 tended to be younger than patients with ST1, ST48, ST117, or this cohort overall (median (IQR) age for ST23 was 62 (50-73) years versus 65 (55-79) years for ST1, versus 70 (57-77) years for ST48, versus 66 (53-76) for ST 117 and versus 67 (55-77) years for entire cohort ( $p=0.29,0.08,0.21$, 0.15 , respectively)). Deaths during follow-up were not significantly higher among patients with ST23 $(\mathrm{n}=14 / 32,43.2 \%)$ than for ST1 $(\mathrm{n}=26 / 88,29.5 \%)$, for ST48 $(\mathrm{n}=18 / 60,30.0 \%)$, for ST117 $(\mathrm{n}=16 / 56,28.6 \%)$ or for the cohort overall $(\mathrm{n}=113 / 371,30.5 \%)(\mathrm{p}=0.19,0.25,0.18,0.16$, respectively).

\section{Discussion}

Over recent years, there has been a large increase in the number of cases of MAB infections worldwide, and the reasons for this are poorly understood [1-3, 31,32]. MLST typing enables a better understanding of the epidemiology of $\mathrm{MAB}$ and helps to establish whether there are major changes in the population structure of clinically relevant MAB. Despite the frequency of reports of MAB colonising and infecting lungs in the largely non-CF populations in Asia, molecular epidemiology studies are scarce in this region [11, 23-25].

The preliminary findings of this study are twofold: first, ST23 and the genetically related, ST48 of clonal cluster 3 (CC3) are clinically nascent in non-CF patients in Taiwan, second and more disconcertingly, is

\section{TABLE 3 Mycobacterium abscessus sequence types over consecutive years from 2010 to 2017 in Taiwan.}

\begin{tabular}{|c|c|c|c|c|c|c|c|c|}
\hline & 2010 & 2011 & 2012 & 2013 & 2014 & 2015 & 2016 & 2017 \\
\hline Total no. of MAB isolates & 35 & 34 & 38 & 35 & 34 & 42 & 42 & 40 \\
\hline ST1 & $28.6(10)$ & 26.5 (9) & 23.7 (9) & $22.9(8)$ & $23.5(8)$ & $23.8(10)$ & $11.9(5)$ & $22.5(9)$ \\
\hline ST48 & $8.6(3)$ & $17.6(6)$ & $26.3(10)$ & $11.4(4)$ & $23.5(8)$ & $16.7(7)$ & $19.0(8)$ & $17.5(7)$ \\
\hline ST117 & $22.9(8)$ & $14.7(5)$ & $15.8(6)$ & $14.3(5)$ & $11.8(4)$ & $16.7(7)$ & $16.7(7)$ & $5.0(2)$ \\
\hline Other STs & $34.3(12)$ & $35.3(12)$ & $31.6(12)$ & $40.0(14)$ & $38.2(13)$ & $42.8(18)$ & 45.2 (19) & 32.5 (13) \\
\hline
\end{tabular}

Data are presented as \% (n), unless otherwise states. Tri-Service General Hospital, Taipei Veterans General Hospital and NTUH-HC could not be included in the longitudinal analysis since they did not submit isolates preceding 2016. MAB: Mycobacterium abscessus; ST: sequence type. 
FIGURE 4 Trends in relative frequencies of the four predominant Mycobacterium abscessus sequence types (STs) between 2010-2017 across Taiwan. Sites Tri-Service General Hospital, Taichung Veterans' General Hospital and National Taiwan University Hospital Hsinchu could not be included in the longitudinal analysis since they did not submit isolates preceding 2016.

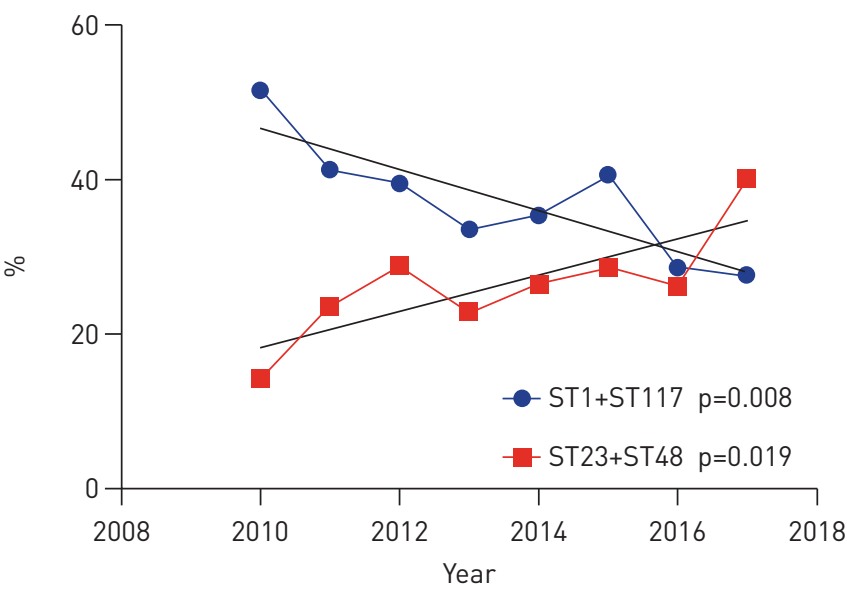

that this globally successful CC3, might be increasing, in contrast to the declining dominance of more genetically diverse ST1 and ST117. The observation that CC3 clones appear to spread among clinical environments without a specific common underlying host immune defect such as CF or cytokine blockade by autoantibody or therapy, may contribute to the increasing incidence of human infections. Our study also highlight that current recommendations for infection control policies to be implemented to minimise risks of person-to-person transmission of $\mathrm{MAB}$ in $\mathrm{CF}$ clinics may be misdirected for two reasons: one, for limiting these policies to CF populations, two, for blanket treatment of all MAB, when the threat may lie specifically with CC3 [33].

Our cohort featured a predominance of patients with head and neck squamous cell carcinoma, lung and oesophageal cancer patients and those requiring tracheotomy for functional disorders such as advanced Parkinson's disease, amyotrophic lateral sclerosis, post-stroke vocal cord or bulbar palsies and dysphagia that accompanies neurodegenerative diseases such as senile dementia. Anatomical or functional abnormalities of the upper aerodigestive tract might be an underappreciated risk factor for $\mathrm{MAB}$ pulmonary disease. $\mathrm{MAB}$ has been demonstrated in $74 \%$ of patients with gastric symptoms in their biopsied gastric epithelium [34]. More studies are warranted to quantify the risks of such upper aerodigestive tract abnormalities.

Although microdroplet nuclei aerosolised after an individual coughs, with subsequent inhalation by an uninfected host, is not an inferred mode of spread of MAB, this possibility has not been excluded, specifically for CC3 [19]. ST23 is the only MAB so far reported to be unusually conserved in widespread outbreaks without a specific or linked environmental source $[18,19]$. Previous studies using pathogenomic analyses suggests $\mathrm{MAB}$ share features such as cording, a virulence associated phenomenon, with $M$. tuberculosis [35]. However, the ST of the MAB strains in these biological studies and in other clinical or environmental surveillance reports are not known [36, 37].

To our knowledge, this is the largest longitudinal molecular epidemiological study conducted of MAB pulmonary isolates. The previous largest collection of MAB reported in a cross-sectional manner was used to establish the international MLST database, and comprised 227 isolates including both pulmonary and soft tissue isolates, a large proportion of which were obtained from French and CF patients, from which 100 STs and 11 clonal clusters or complexes were identified [21]. Subsequent studies employing this MLST scheme are summarised in table 4, including one small study conducted in Ireland of 36 isolates obtained between 2006-2012 from 36 patients, 18 of whom had CF [30] and the other in Scotland of 178 strains sequentially isolated from 12 patients, 10 of whom had CF [38]. The only small single-centre study typing 55 isolates from 55 patients with pulmonary MAB in a non-CF cohort was conducted in Shanghai [39]. In all the above studies, ST23 was detected more than once, but ST48 was found only once in the Shanghai study and not at all in the European studies [30, 38, 39]. Although ST23 (5 of 26 isolates) and not ST48 (1 of 26) was the most prevalent MAB-m in the Shanghai study, the second most common MAB-m was also ST117 (2 of 26 isolates). At the time of publication, 7 of 36 (19\%) and 32 of 55 (58\%) strains in the Irish and mainland Chinese study were novel STs. On an individual level, ST23 has been shown to replace a MAB-a strain (ST122) in 1 of 12 patients studied longitudinally, while the remaining 11 were persistently colonised with the same ST over years [38].

The major limitation of this study was the lack of WGS and access to the previously reported Seattle/ Papworth outbreak strains, to confirm the discriminatory power of MLST. Nevertheless, MLST as previously used by TETTELIN et al. [18] to cluster MAB-m outbreak strains, has been validated by WGS [19]. In addition, MLST as used by TETTELIN et al. [18] has been shown to be in high agreement with 
TABLE 4 Published molecular epidemiological studies using the Pasteur Institute's International MLST database of Mycobacterium abscessus pulmonary isolates from different countries

\begin{tabular}{|c|c|c|c|c|c|c|c|c|}
\hline Year, country & $\begin{array}{l}\text { No. of isolates } \\
\text { (patients) }\end{array}$ & $\begin{array}{c}\text { Cystic } \\
\text { fibrosis \% }\end{array}$ & MAB-a \% & MAB-b \% & MAB-m \% & Dominant STs \% & Novel STs \% & $\begin{array}{l}\text { Singleton } \\
\text { STs } \%\end{array}$ \\
\hline 1998-2010, Scotland [35] & $178(12)$ & 83 & 38 & 16 & 46 & ST23 (42) & 50 & 67 \\
\hline $\begin{array}{l}\text { 2010-2017 Taiwan } \\
\text { (present study) }\end{array}$ & $371(371)$ & 0 & 49 & 1 & 50 & $\begin{array}{c}\text { ST1 (24) } \\
\text { ST48 (16) } \\
\text { ST117 (15) } \\
\text { ST23 (9) }\end{array}$ & 16 & 13 \\
\hline
\end{tabular}

MLST: multilocus sequence typing; MAB-a: M. abscessus subsp. abscessus; MAB-b: M. abscessus subsp. bolletii; MAB-m: M. abscessus subsp. massiliense; ST: sequence type; NA: not available.

the MLST scheme used here [40]. As MLST is more accessible than WGS to the developing world, and the MLST scheme used here is publicly available on the Pasteur Institute's database unlike the scheme of Tettelin et al. [18], most studies conducted from 2014 onwards have used this scheme (table 4) [40].

Given that Taiwan is an island and that our population has one of the lowest incidences of CF worldwide, it is not surprising that a fair number of our isolates were novel STs. By the same token, ST48 was uniquely more common than ST23 in Taiwan. This finding challenges the assumption that ST23 is the common ancestor of CC3, but due to the predominance of ST23 over ST48 in mainland China and in Europe, ST23 may have recently been imported into southern Taiwan, where the largest trade harbour, the Port of Kaohsiung accounting for an annual volume of more than 10 million TEU, is located.

One might also expect individual MAB-a strain prevalence in Taiwan to differ from other countries, given its isolation as an island. However, like previously published studies conducted in Europe, ST1 remains the most common isolate overall in our study and the diversity of MAB-a isolates exceeded that of MAB-m $[21,30]$. While it has been suggested that Asians possess an undefined genetic susceptibility to MAB infection, and our previous outbreak of extrapulmonary post-procedural infections was also due to ST48, whether ST48 is indeed more common or specific among Asian people compared to Caucasian or Hispanic people, remains to be determined. More studies from other continents such as Australia and Africa using this MLST scheme rather than WGS, which may be too cost-prohibitive, are necessary to clarify whether only ST23 (or all members of CC3) should be highlighted for relative evolutionary deviations from traditional MAB and preventative infection control measures.

In conclusion, this population-level, longitudinal molecular epidemiology study documents a possible recent decline in the traditionally dominant and diverse ST1 of MAB-a and increasing recognition of genetically related, MAB-m clones. As reported in outbreaks and non-outbreak studies among CF in the United States, United Kingdom, France, Ireland, Germany, Switzerland and Brazil, CC3 appears to be increasingly clinically prevalent among respiratory isolates of non-CF patients in Taiwan. These findings are likely to be explained by the environmental prevalence of CC3 that are more likely to infect humans, perhaps associated with their exceptional resistance to disinfectants such as alcoholic chlorhexidine and povidone iodine [41]. Novel features from this study include the prominence of ST48, which is closely related to ST23 and assigned to the same clonal complex 3, and the lower percentage of singleton isolates with new MLST types compared to the earlier MLST studies. Further global epidemiology using a shared common molecular language are needed to understand the evolution and dissemination of potentially "fitter" clones of MAB.

Acknowledgements: We thank Yu-Chung Chuang of the National Taiwan University College of Medicine for statistical review. We thank all Laboratory Medicine department staff for storage and access to the mycobacterial isolates. 
Author contributions: A. Cheng conceived and designed the study, coordinated the collection of mycobacterial isolates, analysed the results, and wrote the manuscript. H-Y. Sun designed the study, helped collect mycobacterial results and reviewed the manuscript. Y-T. Tsai conducted the experiments, drew the figures and analysed the results. P-L. Liu, S.S-J. Lee, Y-T. Lee, Y-C. Wang, P-Y. Liu, J-Y. Chien, P-R. Hsueh and S-Y. Chang helped collect the mycobacterial isolates and patient data, and execute the study, and reviewed the manuscript. U-I. Wu and W-H. Sheng reviewed the manuscript. Y-C. Chen and S-C. Chang hosted the study, analysed the results and reviewed the manuscript.

Conflict of interest: None declared.

Support statement: This study was funded by Taiwan's Ministry of Science and Technology (grant number 105-2628-B-002-019-MY3) and the Taiwan's Ministry of Health and Welfare (MOHW107-521 TDU-B-211-113002). Funding information for this article has been deposited with the Crossref Funder Registry.

\section{References}

1 Benwill JL, Wallace RJ Jr. Mycobacterium abscessus: challenges in diagnosis and treatment. Curr Opin Infect Dis 2014; 27: 506-510.

2 Ryan K, Byrd TF. Mycobacterium abscessus: shapeshifter of the mycobacterial world. Front Microbiol 2018; 9 2642.

3 Lopeman RC, Harrison J, Desai M, et al. Mycobacterium abscessus: environmental bacterium turned clinical nightmare. Microorganisms 2019; 7: 90.

4 Leao SC, Tortoli E, Viana-Niero C, et al. Characterization of mycobacteria from a major Brazilian outbreak suggests that revision of the taxonomic status of members of the Mycobacterium chelonae-M. abscessus group is needed. J Clin Microbiol 2009; 47: 2691-2698.

5 Leao SC, Tortoli E, Euzéby JP, et al. Proposal that Mycobacterium massiliense and Mycobacterium bolletii be united and reclassified as Mycobacterium abscessus subsp. bolletii comb. nov., designation of Mycobacterium abscessus subsp. abscessus subsp. nov. and emended description of Mycobacterium abscessus. Int J Syst Evol Microbiol 2011; 61(Pt 9): 2311-2313.

6 Tortoli E, Kohl TA, Brown-Elliott BA, et al. Emended description of Mycobacterium abscessus, Mycobacterium abscessus subsp. abscessus and Mycobacterium abscessus subsp. bolletii and designation of Mycobacterium abscessus subsp. massiliense comb. nov. Int J Syst Evol Microbiol 2016; 66: 4471-4479.

7 Adekambi T, Sassi M, van Ingen J, et al. Reinstating Mycobacterium massiliense and Mycobacterium bolletii as species of the Mycobacterium abscessus complex. Int J Syst Evol Microbiol 2017; 67: 2726-2730.

8 Tortoli E, Kohl TA, Brown-Elliott BA, et al. Mycobacterium abscessus, a taxonomic puzzle. Int J Syst Evol Microbiol 2018; 68: 467-469.

9 Lee MC, Sun PL, Wu TL, et al. Antimicrobial resistance in Mycobacterium abscessus complex isolated from patients with skin and soft tissue infections at a tertiary teaching hospital in Taiwan. J Antimicrob Chemother 2017; 72: 2782-2786.

10 Bryant JM, Grogono DM, Greaves D, et al. Whole-genome sequencing to identify transmission of Mycobacterium abscessus between patients with cystic fibrosis: a retrospective cohort study. Lancet 2013; 381: 1551-1560.

11 Koh WJ, Jeon K, Lee NY, et al. Clinical significance of differentiation of Mycobacterium massiliense from Mycobacterium abscessus. Am J Respir Crit Care Med 2011; 183: 405-410.

12 Chang PH, Chuang YC. Anti-interferon-gamma autoantibody-associated disseminated Mycobacterium abscessus infection mimicking parotid cancer with multiple metastases: a case report. Medicine (Baltimore) 2017; 96: e8118.

13 Hong GH, Chetchotisakd P, Anunnatsiri S, et al. Natural history of anti-interferon-gamma autoantibody-associated immunodeficiency syndrome in Thailand. J Clin Immunol 2019; 39: S51-S51.

14 Aoki A, Sakagami T, Yoshizawa K, et al. Autoimmune nontuberculous mycobacterial disease: characteristic phenotypes of cases with anti-interferon-gamma neutralizing autoantibodies. Am J Respir Crit Care Med 2017; 195: A5065.

15 Bange FC, Brown BA, Smaczny C, et al. Lack of transmission of mycobacterium abscessus among patients with cystic fibrosis attending a single clinic. Clin Infect Dis 2001; 32: 1648-1650.

16 Harris KA, Underwood A, Kenna DT, et al. Whole-genome sequencing and epidemiological analysis do not provide evidence for cross-transmission of Mycobacterium abscessus in a cohort of pediatric cystic fibrosis patients. Clin Infect Dis 2015; 60: 1007-1016.

17 Aitken ML, Limaye A, Pottinger P, et al. Respiratory outbreak of Mycobacterium abscessus subspecies massiliense in a lung transplant and cystic fibrosis center. Am J Respir Crit Care Med 2012; 185: 231-232.

18 Tettelin H, Davidson RM, Agrawal S, et al. High-level relatedness among Mycobacterium abscessus subsp. massiliense strains from widely separated outbreaks. Emerg Infect Dis 2014; 20: 364-371.

19 Bryant JM, Grogono DM, Rodriguez-Rincon D, et al. Emergence and spread of a human-transmissible multidrug-resistant nontuberculous mycobacterium. Science 2016; 354: 751-757.

20 Nunes LDS, Baethgen LF, Ribeiro MO, et al. Outbreaks due to Mycobacterium abscessus subsp. bolletii in southern Brazil: persistence of a single clone from 2007 to 2011. J Med Microbiol 2014; 63(Pt 10): 1288-1293.

21 Macheras E, Konjek J, Roux AL, et al. Multilocus sequence typing scheme for the Mycobacterium abscessus complex. Res Microbiol 2014; 165: 82-90.

22 Cheng A, Sheng WH, Huang YC, et al. Prolonged postprocedural outbreak of Mycobacterium massiliense infections associated with ultrasound transmission gel. Clin Microbiol Infect 2016; 22: 382 e1-382 e11.

23 Lim AYH, Chotirmall SH, Fok ETK, et al. Profiling non-tuberculous mycobacteria in an Asian setting: characteristics and clinical outcomes of hospitalized patients in Singapore. BMC Pulm Med 2018; 18: 85.

24 Nagano H, Kinjo T, Nei Y, et al. Causative species of nontuberculous mycobacterial lung disease and comparative investigation on clinical features of Mycobacterium abscessus complex disease: A retrospective analysis for two major hospitals in a subtropical region of Japan. PLos ONE 2017; 12: e0186826.

25 Huang HL, Cheng MH, Lu PL, et al. Epidemiology and predictors of NTM pulmonary infection in Taiwan - a retrospective, five-year multicenter study. Sci Rep 2017; 7: 16300. 
Wu M, Li B, Guo Q, et al. Detection and molecular characterization of amikacin-resistant Mycobacterium abscessus isolated from patients with pulmonary disease. J Glob Antimicrob Resist 2019; 19: 188-191.

Cheng A, Liu YC, Chen ML, et al. Extrapulmonary infections caused by a dominant strain of Mycobacterium massiliense (Mycobacterium abscessus subspecies bolletii). Clin Microbiol Infect 2013; 19: E473-E482.

Zelazny AM, Root JM, Shea YR, et al. Cohort study of molecular identification and typing of Mycobacterium abscessus, Mycobacterium massiliense, and Mycobacterium bolletii. J Clin Microbiol 2009; 47: 1985-1995.

Griffith DE, Aksamit T, Brown-Elliott BA, et al. An official ATS/IDSA statement: diagnosis, treatment, and prevention of nontuberculous mycobacterial diseases. Am J Respir Crit Care Med 2007; 175: 367-416.

O’Driscoll C, Konjek J, Heym B, et al. Molecular epidemiology of Mycobacterium abscessus complex isolates in Ireland. J Cyst Fibros 2016; 15: 179-185.

Chien JY, Lai CC, Sheng WH, et al. Pulmonary infection and colonization with nontuberculous mycobacteria, Taiwan, 2000-2012. Emerg Infect Dis 2014; 20: 1382-1385.

Prevots DR, Marras TK. Epidemiology of human pulmonary infection with nontuberculous mycobacteria: a review. Clin Chest Med 2015; 36: 13-34.

Haworth CS, Banks J, Capstick T, et al. British Thoracic Society Guideline for the management of non-tuberculous mycobacterial pulmonary disease (NTM-PD). BMJ Open Respir Res 2017; 4: e000242.

Chouhan D, Barani Devi T, Chattopadhyay S, et al. Mycobacterium abscessus infection in the stomach of patients with various gastric symptoms. PLoS Negl Trop Dis 2019; 13: e0007799.

Howard ST. Recent progress towards understanding genetic variation in the Mycobacterium abscessus complex. Tuberculosis (Edinb) 2013; 93 Suppl: S15-S20.

Bernut A, Herrmann JL, Kissa K, et al. Mycobacterium abscessus cording prevents phagocytosis and promotes abscess formation. Proc Natl Acad Sci USA 2014; 111: E943-E952.

Kuo YM, Cheng A, Wu PC, et al. Disseminated Mycobacterium abscessus infection and showerheads, Taiwan. Emerg Infect Dis 2011; 17: 2077-2078.

8 Kreutzfeldt KM, McAdam PR, Claxton P, et al. Molecular longitudinal tracking of Mycobacterium abscessus spp. during chronic infection of the human lung. PLoS ONE 2013; 8: e63237.

9 Luo L, Li B, Chu H, et al. Characterization of Mycobacterium abscessus subtypes in Shanghai of China: drug sensitivity and bacterial epidemicity as well as clinical manifestations. Medicine (Baltimore) 2016; 95: e2338.

Cheng A, Sun HY, Tsai YT, et al. Comparing the utilities of different multilocus sequence typing schemes for identifying outbreak strains of Mycobacterium abscessus subsp. massiliense. J Clin Microbiol 2019; 58: e01304-19.

Cheng A, Sun HY, Tsai YT, et al. In vitro evaluation of povidone-iodine and chlorhexidine against outbreak an nonoutbreak strains of Mycobacterium abscessus using standard quantitative suspension and carrier testing. Antimicrob Agents Chemother 2018; 62: e01364-17. 